\title{
Writing Case Reports: Teaching and Tuition Techniques, and the Improvement of Clinical Diagnostic Reasoning
}

Yosuke Kakisaka, 1,2 Mayu Fujikawa,' Schuyler Gaillard. ${ }^{3}$

About the Author: Yosuke Kakisaka is a pediatrician and epileptologist at Tohoku University Graduate school of Medicine in Sendai, Japan.
The case report is a time-honored, important, integral, and widely accepted component of the medical literature.' Reporting of cases illustrating new or rare clinical phenomena is highly encouraged to extend medical knowledge through patient treatment. The process of writing a case report is essential to medicine and patient care, as described by 0sler.

\section{Writing Case Reports Enhances Clinical Diag- nostic Reasoning \\ We present two example cases which illustrate how writing case reports enhances clinical diagnostic reasoning. The first important case report of author YK described a patient en- countered during a pediatric residency over 10 years ago. A 13-year-old girl visited a pediatric outpatient clinic. Her comp- laint was periodic periumbilical abdominal pain with episodes lasting several hours. This symptom was stereotypical between episodes and remitted spontaneously every time. However, routine medical examination was unremarkable. The process of exploring the pathophysiology of an abdominal pain throu- gh literature search was tremendously intriguing. Several case reports indicated abdominal pain as one of the various extra- cephalic symptoms of migraine. The patient was finally diag- nosed with abdominal migraine, a migraine variant, and her abdominal pain was relieved with sumatriptan administration. This case was published in the Japanese literature. ${ }^{3}$ This expe- rience was the departure point of YK's exploration of the cu- rious pathophysiology of migraine and its variants. Since then, this rare extracephalic symptom has been added to the list of screening symptoms for patients complaining of headaches during history taking.}

YK subsequently encountered the case of a 34 -year-old female with a family history of migraines. She was referred to our clinic for evaluation of recurrent epigastric pain with severe cramping, which had started at age 9 years with a frequency of once per month. Gastrointestinal evaluation was performed between attacks and found no abnormality. The patient also complained of severe throbbing headaches lateralized to the right temporal region, which appeared at age 24 years. These headaches lasted for a maximum of several days per episode, with a frequency of once per month. The patient had a prior diagnosis of temporal lobe epilepsy at a previous hospital based on electroencephalography indicating temporal intermittent rhythmic delta activity in the left hemisphere without obvious spikes. However, YK's initial assessment suspected abdominal migraine because of her abdominal symptoms and long duration of intense pain. In contrast, mesial temporal lobe epilepsy typically presents with short epigastric discomfort. The coexistence of cephalic migraines also supported the diagnosis of abdominal migraine. Further investigation of her epigastric attacks revealed the presence of photophobia and phonophobia during her abdominal pain episodes. Consequently, she was diagnosed with abdominal migraine. Sumatriptan administration successfully controlled the abdominal pain episodes. ${ }^{4}$ Previous experience of researching and summarizing knowledge of abdominal migraine, undertaken for the aforementioned case report, 3 facilitated the correct diagnosis.

Clinical diagnostic reasoning is defined as "the thinking process to reach the appropriate conclusions for diagnosis and treatment." 5 Clinical diagnostic reasoning is based on fundamental factors: knowledge about disease, context of patient's history, and clinical experience. ${ }^{5}$ All of these aspects are essential in case writing. Figure 1 describes a theoretical model of the relationship between clinical diagnostic reasoning and case writing. The first step of clinical diagnostic reasoning is acquiring clinical information based on the patient's history (shaded box in Figure 1). The second step is to understand the represented problem, which is the process of formulating a hypothesis. To reach the most appropriate diagnosis from the hypothesis, search for the defining and discriminating features of each possible disease under consideration with additional targeted data gathering and literature search.

The process of case writing necessitates thorough investigation of the context of patient history, in addition to increasing clinical experience by reviewing clinical and imaging data. Furthermore, case writing requires analysis of the literature that describes similar symptoms. Both clinical interactions and literature review increase specific disease knowledge. Therefore, case writing provides the opportunity to combine knowledge about disease, the context of the patient history, and clinical experience. Therefore, we consider that publishing case reports (about disease $X$ ) enhances the physician's clinical diagnostic reasoning skills (about disease $X$ ), which will be helpful in following further cases (of disease $\mathrm{X}$ ). In the following section,

Department of Epileptology, Tohoku University Graduate School of Medicine, Sendai, Miyagi, Japan.

2 Department of Pediatrics, Tohoku University Graduate School of Medicine, Sendai, Miyagi, Japan.

Department of Brain and Cognitive Sciences, Massachusetts Institute of Technology, Cambridge, MA, USA.

Correspondence:

Yosuke Kakisaka.

Address: Department of Epileptology, Tohoku University Graduate School of Medicine, 2-1 Seiryo-machi, Aoba-ku, Sendai, Miyagi 980-8575, Japan.

Email: kakisuke@mui.biglobe.ne.jp 


\section{Editorial}

we introduce the theoretical process of case writing and how it may benefit clinical diagnostic reasoning.

\section{Writing Case Reports as Medical Education}

We recently launched an educational case writing program for medical students at Tohoku University School of Medicine. The program consists of two parts: (1) a didactic lecture for learning the principles of case writing, and (2) a hands-on case writing practice to apply the learned skills in a clinical case. The didactic lecture focuses on identifying the topic for a case report. A desirable topic should include both scientific significance and novel knowledge illustrated by the report. The topic may describe the patient's symptoms (e.g. clinical course, imaging), utility of a medical modality, or even specific clinical actions (e.g. hints for efficacious history taking). We recommend three steps to identify the topic for a case report: (1) to read related case reports in order to increase knowledge of the patient's condition; (2) to make a table to compare the current patient's symptoms with previously described symptoms; and (3) to critically assess whether the topic has reportable significance as a case report. The details and educational efficacy of the didactic part of this program are presented separately in a Letter to the Editor published in this issue of International Journal of Medical Students (IJMS). ${ }^{6}$

Students in the program write case reports under the supervision of faculty staff in the second part of the program. Figure 2 illustrates the developmental course of writing skill through the program. After the lecture on the principles of case writing, the students begin writing actual case reports. Initial drafts produced by the students typically need drastic correction. Brainstorming or Socratic questioning is often effective in enhancing students critical thinking, through questions such as "what is the main topic of this case?", "how can we describe it scientifically?", and "how should we form a hypothesis of the mechanism under discussion?" Multiple discussion sessions between students and faculty increase the quality of the manuscript, which in turn improves case writing skills and the level of theoretical understanding about the case writing principles. Each subsequent draft should require less guidance from the faculty, and the students will eventually achieve a satisfactory skill level (n-th paper of Figure 2).

Medical students often struggle to reach the correct diagnosis based on clinical diagnostic reasoning because they often lack the three skill factors (knowledge of disease, context of patient's history, and clinical experience). We believe that multiple experiences with writing case reports will help improve the knowledge of clinical diagnostic reasoning among medical students. In particular, once a medical student publishes a case report, the sense of accomplishment will bring pleasure and reward for the finished case report and motivate the student to undertake the task of writing another case report.?

In the future, the educational case writing program may allow wider opportunities to include medical students in distant or rural areas with limited opportunity for writing scientific papers. Using a high-definition telemedicine system, students interested in learning about case report writing can access our program with face-to-face tuition. To promote this program to students in remote areas, we have started to provide lectures
Figure 1. Proposed Modification of Bowen's Model of Key Elements of Clinical Diagnostic Reasoning 5

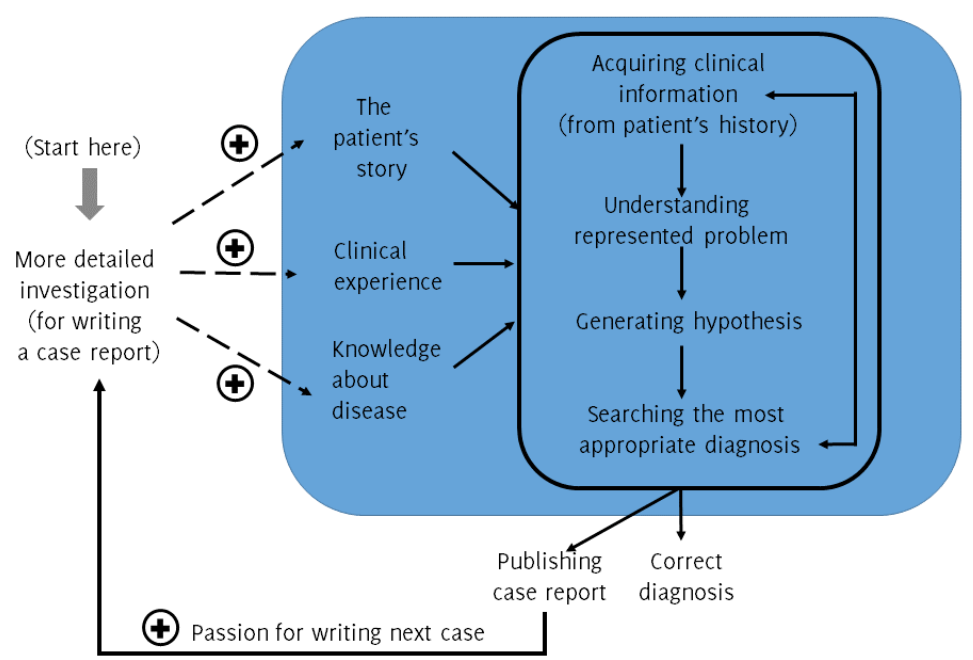

Dashed arrows indicate the potential effects of writing and publishing case reports about disease $\mathrm{X}$ on clinical diagnostic reasoning applied to subsequent cases of disease $X$.

Figure 2. Feedback in the Process of Improving Skills

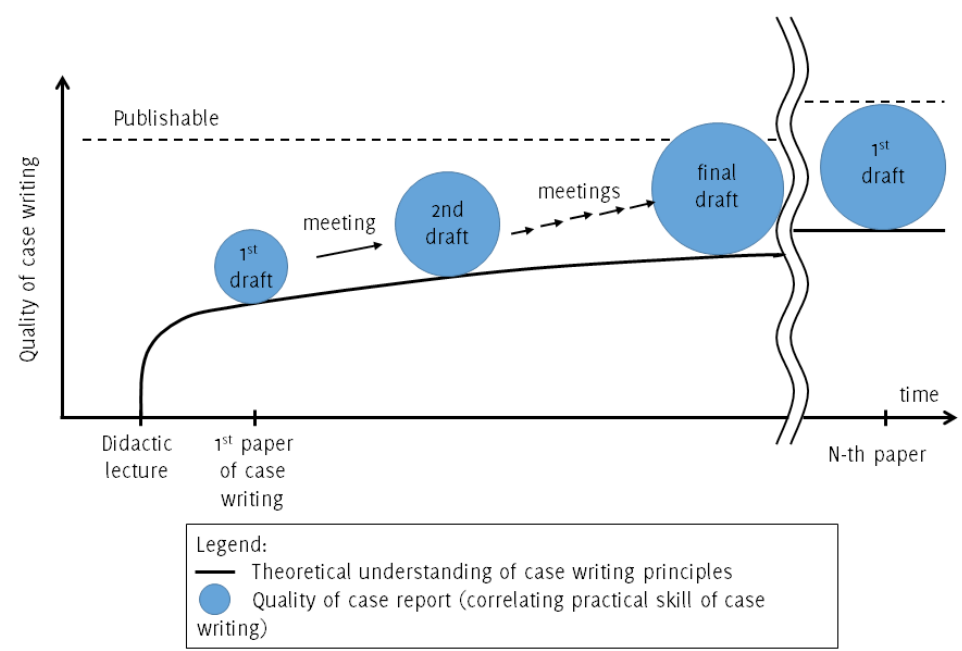

After the didactic lecture (step 1) about the principles of case writing, students begin the process of actual case writing. Initially, drafts are immature even after receiving the lecture, as both theoretical understanding and practical skills of case writing lack experience. Multiple meetings between the student and advisor increase the quality of the current case report, which correlates with the practical skills of case writing, as well as increase the theoretical understanding of case writing principles. Therefore, the quality of the first draft of the $n$-th paper will be higher than that of the first paper.

and discussion via the telemedicine system. Integration of such new teaching techniques and developed technologies may establish a new approach to medical education. 


\section{References}

1. Ortega-Loubon C, Correa-Marquez R. Writing a case report: a work of art. Int J Med Students. 2014 Jul-0ct;2(3):90-1.

2. Thayer WS. Osler, the teacher. In: Sir William Osler, Bart. Baltimore: Johns Hopkins Press; 1920. p. 51-2.

3. Kakisaka Y, Matsuki S, Miura Y, Nakayama S, Sato T, Tanabu M, et al. [Successful treatment with sumatriptan in a case with abdominal migraine]. Japanese J Clin Pediatr. 2004 Nov;57(11):2285-9. Japanese

4. Kakisaka $\mathrm{Y}$, Jin $\mathrm{K}$, Kato $\mathrm{K}$, Iwasaki M, Nakasato N. Temporal intermittent rhythmic delta activity and abdominal migraine. Neurol Sci. 2014 Apr;35(4):627-8.

5. Bowen JL. Educational strategies to promote clinical diagnostic reasoning. N Engl J Med. 2006 Nov 23;355(21):2117-25.

6. Kakisaka Y. Structured didactic education program for writing case reports can motivate medical students. Int J Med Students. 2016 Sep-Dec;4(3): Forthcoming.

7. Petrusa ER, Weiss GB. Writing case reports: an educationally valuable experience for house officers. J Med Educ. 1982 May;57(5):415-7.

\section{Acknowledgments}

We would like to thank Francisco Javier Bonilla-Escobar, Editor-in-Chief of IJMS, for the invitation to write an Editorial to the Journal regarding our experience on writing case reports.

Conflict of Interest Statement at Funding

The authors have no funding, financial relationships, or conflicts of interest to disclose.

\section{Author Contributions}

Conceptualization: YK. Writing, Critical revision of the manuscript, Approval of the final version: YK, MF, SG.

Cite as:

Kakisaka Y, Fujikawa M, Gaillard S. Writing case reports: teaching and tuition techniques, and the improvement of clinical diagnostic reasoning. Int J Med Students. 2016 Sep-Dec;4(3):88-90. 